\title{
,ance \\ Clash Cost Analysis in Electrical Installations Based on BIM Technologies
}

\author{
Tadeusz Daszczyński ${ }^{1, *(D)}$, Michał Ostapowski ${ }^{1}$ and Aleksander Szerner ${ }^{2}$ \\ 1 Department of Electrical Engineering, Institute of Electrical Power Engineering, \\ Warsaw University of Technology, 00-662 Warsaw, Poland; michal.ostapowski@gmail.com \\ 2 BIM Association, 02-316 Warsaw, Poland; aleksander.szerner@plbim.org \\ * Correspondence: tadeusz.daszczynski@pw.edu.pl
}

check for updates

Citation: Daszczyński, T.;

Ostapowski, M.; Szerner, A. Clash

Cost Analysis in Electrical

Installations Based on BIM

Technologies. Energies 2022, 15, 1679.

https://doi.org/10.3390/en15051679

Academic Editors: Fahim Ullah and Paulo Santos

Received: 13 January 2022

Accepted: 22 February 2022

Published: 24 February 2022

Publisher's Note: MDPI stays neutral with regard to jurisdictional claims in published maps and institutional affiliations.

Copyright: (C) 2022 by the authors. Licensee MDPI, Basel, Switzerland. This article is an open access article distributed under the terms and conditions of the Creative Commons Attribution (CC BY) license (https:// creativecommons.org/licenses/by/ $4.0 /)$.

\begin{abstract}
One of the core features of delivering a Building Information Modelling (BIM) project is design coordination and clash detection. In the traditional way of conducting investments, the design process consists in the separate work of individual industry specialists on an individual part of the project and checking its compliance with coordination checkpoints. Building information modelling/management is emerging as an innovative way to virtually design and manage projects. Clashes are one of the most problematic elements in construction. They can interfere in the decisionmaking process by generating unexpected costs of additional materials, work and actions. The purpose of the following paper is to analyze the impact of BIM on limiting cost of clashes and also to check how clashes affect the unforeseen costs of a construction investment and how the BIM methodology can help to reduce them, especially in electrical installations. Basic construction collisions with algorithms of their solutions were presented. The possible financial benefits associated with the use of the BIM approach have been described using mathematical relationships.
\end{abstract}

Keywords: clash analysis; AEC; BIM; cost analysis; electrical installations

\section{Introduction}

There are a few different definitions of Building Information Modelling (BIM); different between investors and contractors and architects and engineers, for their perceptions of BIM and mainly focusing on their differences rather than similarities [1]. One of them is "an intelligent 3D virtual building model that can be constructed digitally-by containing all aspects of building information-into an intelligent format that can be used to develop optimized building solutions with reduced risk and increase value before committing to a design proposal" [2]. This definition is focuses mainly on the design perspective, which will be used for the purpose of this paper.

The last four decades have seen a dynamic development of information technologies. This is especially evident in one of the most important branches of IT-ICT. Computers, the Internet, and the free flow of information push economic progress forward, reducing costs and production time. Progressing automation and computerization have had a positive impact on a large part of the manufacturing industry. This resulted in a significant increase in the productivity of many industries.

Multi-branch building projects are, due to the use by the users of modern technologies (PropTech), more and more complicated and difficult to manage [3]. Proptech can be described as application of technology in the real estate industry value chain. The intention of this technology is to integrate disruptive solutions and enhance productivity. The entire spectrum includes, among others, use of digitization in property management, structure analysis and research, common space management, 3D modeling (BIM), space organization, etc. BIM is currently used to define a modern approach to the entire process of design, construction and operation of buildings, so it is an approach that begins from the moment 
the idea for a building is created until its completion. It is the implementation of a scientific approach to the design process. In this scientific approach the digital model of physical phenomena or the device is created and simulated to check if all the details have been taken into account.

BIM technology integrates various investment aspects including: parameterized geometric model of the object (usually 3D), functional and performance requirements of individual elements, etc. These data can be further used as part of BIM throughout the life cycle of the building for, e.g., creating construction time schedules, construction process management, cost management, maintenance management and property management, etc. The successive steps in the development of BIM technology made it possible to create the concept of a "digital twin" (Figure 1). This digital model of the structure enables smooth data transmission between the physical and virtual worlds and facilitates the monitoring. It helps better understanding and optimization of the functions of all physical units of the facility [4]. To obtain information and data, the digital twin has the ability to download them from all possible sources, including using the BIM model. It also adds the missing data pieces needed during the construction process.

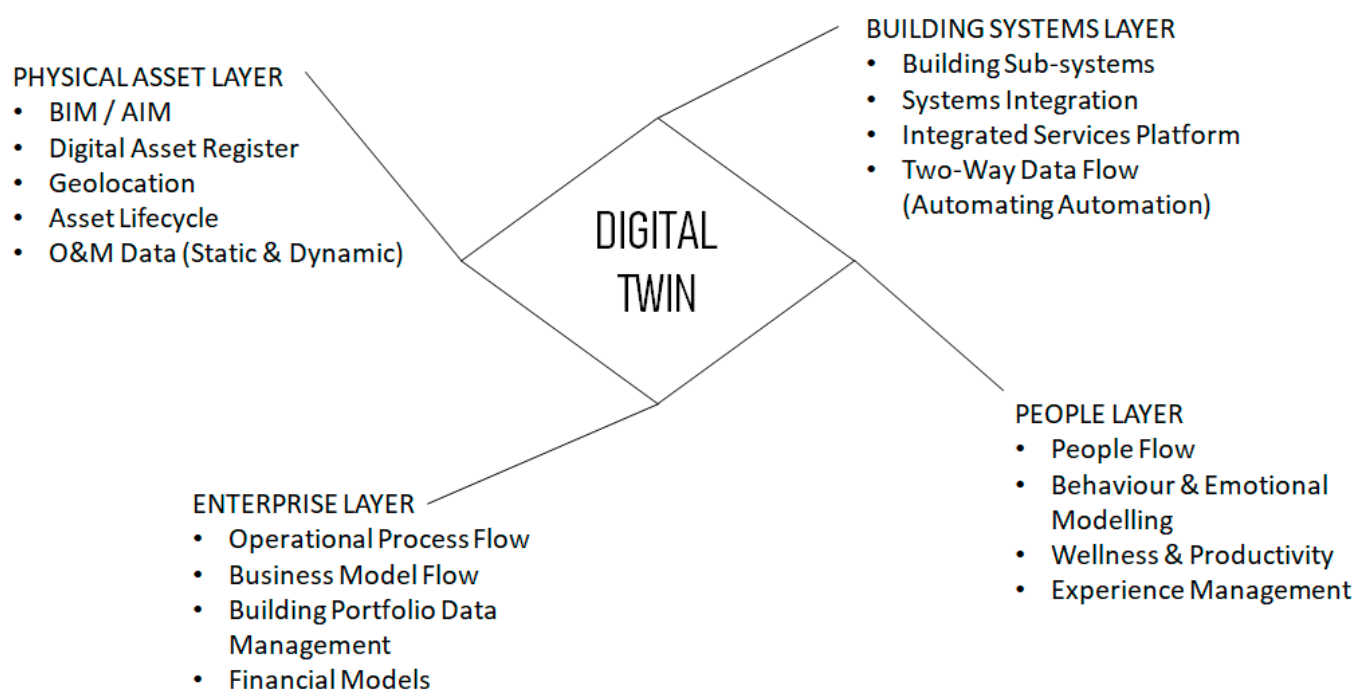

Figure 1. Digital Twin interpretation.

Theoretical developments in BIM suggest that not only is it useful for geometric modelling of a building's performance but also that it can assist in the management of construction projects. There are six main important benefits of BIM:

- $\quad$ Economic and resource savings

- Greater efficiency and shorter project lifecycles

- The use of collaboration in the process

- The use of prefabrication and modular construction

- Robotization and automation

- Higher quality results

The most inspiring factor influencing the development of BIM in Poland (and also probably in the world) is the cost optimization (minimization) factor, taking into account the cost of collisions of different installations. The development of BIM and the study of its impact on costs and benefits is inextricably linked from the very beginning. This is due to the fact that any technical innovation that is to survive in the competitive world of business should have a solid economic basis. In the case of modelling information about a building, research has shown that the main problem has turned out to be the justification of additional costs and benefits. Users seeking to implement BIM need strong incentives based on facts and experience, while investors need to find evidence that will justify the investment of increased time and budget. From a broader perspective, this research can be linked to 
other analyses of the impact of technology on business outcomes, however, measuring the benefits of BIM has its own rules and poses a unique challenge for researchers. Running cable routes in the building area may naturally interfere with e.g., ventilation or sanitary installations. It is also obvious that the electrical installations are more flexible. That is why this aspect is so important and should be raised in the description of the advantages of introducing BIM in electrical installations.

\section{Literature Review}

In design terms a clash occurs when components that make up a built resource are not spatially coordinated and conflict with each other. Clashes can be then described as waste in the production system. Clash detection, even in a traditional 2D investment implementation system is one of many quality checks. It is conducted by designers before the finishing of the project, and they release their drawings or BIM models for further downstream delivery processes. Many clash classifications forms can be found in the literature. Typically, the different approaches refer to impact severity and delay related to the clashes. There are mainly three types of clashes as: hard, soft and time clashes. There is also a different division into major (hard), medium and minor (soft) clashes [5,6] -Figure 2. Another division is made in [7] and it is based on geometrical and time data. It is possible to sort clashes in four types: hard, soft, 4D clashes and animated clashes (clashes of animated objects). In this paper the division shown on Figure 2 is discussed.

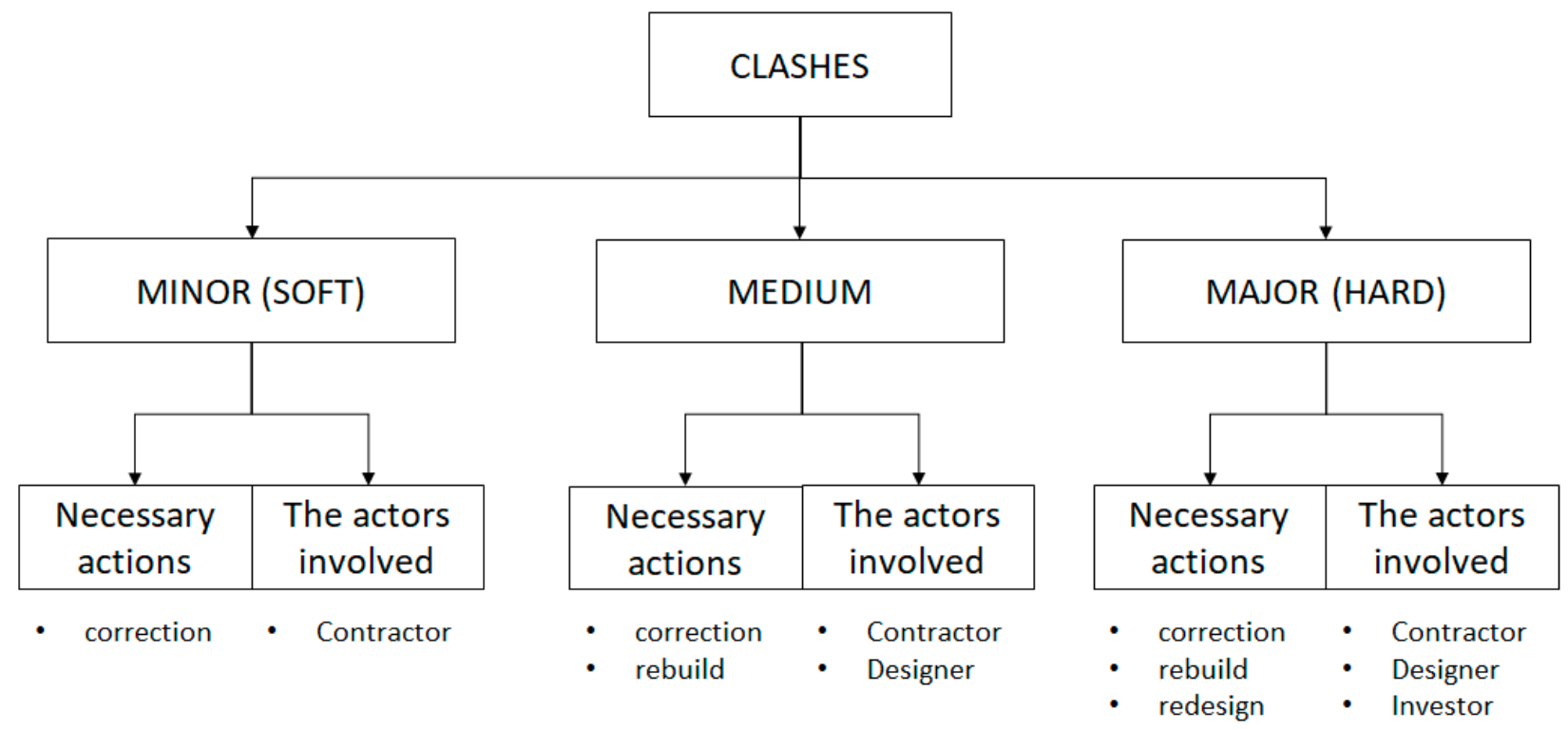

Figure 2. Types of clashes divided into minor (soft), medium and major (hard).

During the traditional design process the specialists are working on separate drawings with tracing papers produced during co-ordination checkpoints to check for compatibility (Figure 3). In this system it is quite usual for clashes to only be spotted on the construction site with the potential of huge costs and delays. During the project stage it was a manual process done by using 2D drawings and looking for potential collisions. The members of a design team (in a traditional design process each designer works alone) must be very experienced in order to prevent design clashes from remaining undetected. 

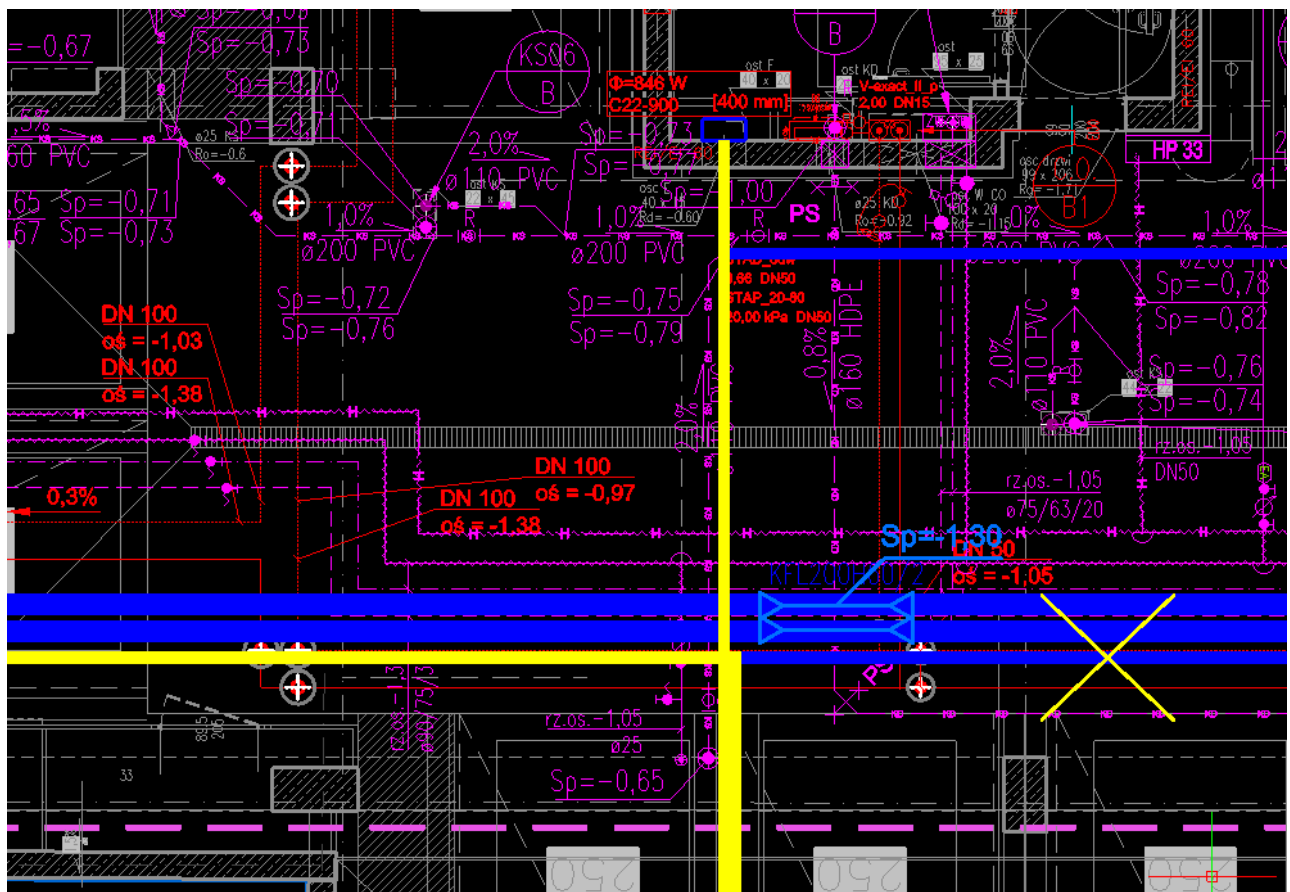

Figure 3. Clash detection during a 2D traditional design process.

Although significant benefits from the use of BIM technology have been demonstrated, the correct and clear justification, e.g., in the form of direct cost savings of implementing BIM in real projects, including collision detection, is not clearly presented in the literature. The project teams can highlight clashes on different level of details. That installation collisions can significantly impact a project especially on the development stage. Finding issues early can reduce total project costs substantially. It has been found that the average cost of clashes (issues on a construction job) are $1500 \$$ per instance [8].

The team's early collaboration is crucial for the final result of the project. The design needs to be optimized and clash-free; this is what automation processes through BIM have the capacity to reduce through 3D design coordination [9].

There are many papers with case studies which show how BIM is effectively used to reduce costs for the General Contractor (GC) of potential collisions or clashes between various structural and mechanical systems. The cost benefits to the owner and GC are significant, and the unknown costs that were avoided were in addition to the savings. Average BIM ROI (i.e., ROI = earning/cost) for projects described in [9] was $634 \%$. This clearly shows its potential economic benefits.

The $4 \mathrm{D}$ structural information model with its main functions was described in $[10,11]$. The integrated solution comprises a kernel information model. All the information required for any function comes from the unique information model. It assumes $4 \mathrm{D}$ schedule conflict management, which the user can do at any time. Schedule conflict analysis can then be conducted in accordance with the relevant project priorities. When any conflicts are detected, the solution, which can be prepared by any involved designer, is able to help managers adjust schedules for follow-up works according to the progress of the project.

During the BIM process some collisions of the mechanical, electrical and plumbing (MEP) systems can be found. Design coordination on a project is the process of checking each party's information against the rest. It is done to ensure a single, unified set of deliverables that achieves the project brief. The design experience of the designers is then mainly applied to solve the clashes [12]:

(1) Gravity driven plumbing system-due to its limited space, adjusting it must first be considered;

(2) HVAC system—due to the large size of components and high price; 
(3) Electrical system with large cross-section cables-due to the lack of flexible cable laying and routing as well as the high price;

(4) Between plumbing, fire protection, control and more mainly due to flexible routing;

(5) Any other rules, such as a small pipe giving way to a big pipe and a cheap component giving way to an expensive component.

The research [13] has shown that BIM is not a complete way to overcome design flaws. The probability density function (PDF) and cumulative distribution function (CDF) probability distribution models are developed to offer complete insight into the size and frequency of clash occurrence. This could be used to develop probability profiles that enable BIM managers to better define and delineate tolerances prior to conducting clash detection. The clash detection report usually contains information such as:

- thematic groupings of clashes;

- $\quad$ snapshots of every clash identified;

- $\quad$ co-ordinates of the collision (as $\mathrm{x}, \mathrm{y}$ and $\mathrm{z}$ coordinates);

- the date;

- clash status (active and unresolved or resolved);

- a written description of the clash;

- a numerical value of the collision.

There can be found data from real construction sites, where the real savings are discussed. During the Leeds Arena project in Great Britain it was found that the BIM clash detection system saved about 350,000 GBP by early identification of possible errors [7]. Holder Construction reported [14] about 135,000 USD savings obtained by BIM clash detection (the system found 35 conflicts) with only 4000 USD in investments in the BIM tools.

The BIM methodology gives the opportunity to create better and more efficient communication and collaboration by all project participants. It is expected to incur not only greater efficiency at the project and construction stage, but also higher quality and speed in decision making, as well as reduced conflicts. Two-thirds of the users state they have seen positive ROI on their investment [15]:

- $87 \%$ of users stated positive ROI

- $\quad 93 \%$ of the users believe that more value can be obtained from it in the future.

Mentioned earlier, Holder Construction decided strategically to integrate BIM for its Aquarium Hilton Garden Inn project. BIM tools identified 55 clashes during the design development stage alone. It resulted in reducing the number of RFIs in the project. As a result, nearly 124,000 USD of the cost of the collision was avoided. The BIM tools expense was 40,000 USD and the savings in the project returned of 84,500 USD. All the tracking of the collisions were made during the construction documentation phase of the model and each time that model was updated. Each time, when the critical clash was detected, it was shared with the design team by means of the model viewer and a numbered collision log. By means of the BIM software over 590 collisions were found. The total cost savings were 800,000 USD [15].

BIM technology provides many and specific opportunities for cost reduction, allowing a significant overall final cost savings for almost any project. The bigger and more complex the project, the greater the opportunity for cost reduction, because of the greater probability of error and change elimination.

\section{Analysis of the Financial Benefits of Using BIM}

Clashes are one of the most problematic elements in construction. They can interfere in the decision making process by generating unexpected costs of additional materials, works and actions. It is impossible to totally eliminate clashes from the construction industry, but it is achievable to limit their side effects, in which case a prompt detection of clashes is a necessity which could be provided by using the BIM methodology.

The importance of the cost analysis of using BIM technology is evidenced by the fact that the European Commission started in 2020 a project to determine the "Methodology 
for cost benefit analysis for the use of BIM in public tenders". The purpose of this contract was [16]:

- developing a model to verify and check the costs and benefits (financial and nonfinancial) of using BIM in public construction projects

- to demonstrate the practical application and validity of a case study model

- development of information material for different public authorities wanting to learn about the cost-benefit analysis of BIM and its application.

The BIM 5D model can only provide data on estimated daily expenses at a construction site. It is not able to show the actual outflow of the contractor's cash, because very often the contracting authority is obliged to make an advance payment on the day the order is placed, while the rest of the fee is paid to the service provider only after the completion of a given part of the work. Therefore, to be able to determine how the expenditure is actually spread out over time, it is necessary to analyze the patterns of fees for equipment, materials and labor [12].

The construction process is characterized by the fact that it requires the use of various types of equipment. Their supply usually involves at least a few suppliers, each of which may be responsible for the provision of various types of equipment, while a given type of equipment may be supplied only by one supplier. For one supplier, the costs of various types of accessories may differ from each other, while the payment periods remain the same. The four factors needed to calculate the cash outflow can be extracted from the BIM model. These are the start, end and working time of construction equipment and their unit price. Full analysis requires additional consideration of the time after which the contractor may at the latest make a payment to the supplier of the equipment.

Payments for labor are made in a monetary unit and are paid at the end of each payment period. The remuneration for each team of employees is calculated on the basis of the number of working days or the number of tasks performed in a given settlement period. Most of the data can be obtained thanks to the BIM model. These are the start, end, and service time of the team of employees, the number of tasks performed/days worked and the unit labor cost of the team. The only external information here is data on payment periods. However, taking into account that the most common settlement period in terms of work is monthly salaries, this factor could also be included in the model.

The construction process involves several $S$ suppliers supplying $L$ of various materials. Each supplier may supply several different materials, but the construction site may be supplied with a given raw material from only one supplier. Each material may be ordered several times, and each order may consist of more than one delivery. At the time of placing the order, the contractor must pay the advance, while the rest is paid after a certain amount of time from the completion of the entire order. In the schemes for calculating cash outflows related to materials, the BIM model can be used to a lesser extent than in the previous cases. Material delivery is a slightly more complex process than renting equipment or hiring employees, and therefore the number of factors that can be predicted in advance is smaller. The 5D model is able to provide in this case only the moment of starting and ending the use of materials and their unit price. The person calculating the balance sheets in this case has a difficult task because he has to take into account as many as seven external variables. These variables are: the moment of payment of the advance and its percentage; the time of order fulfillment; the time that passes from the first delivery to the commencement of use; the time of payment of the remaining amount; and the amount of materials in a given order.

\section{Analysis of Clash Cost in Electrical Installations}

In order to conduct research, clashes were divided into three categories as shown in Figure 2. This categorization was made based on which stakeholders are involved and what actions are obligatory to resolve the clash. Minor conflicts can be independently solved by the contractor because of their low difficulty level. Medium clashes, excepting the impact on the contractor, will require designer approval because of inevitability of 
rebuilding. Major clashes will call for design changes so they will need to be consultations carried out in order to obtain client confirmation.

All the kinds of clashes presented above can influence the construction process in the time and cost domains, wherein these two domains are inseparably connected to each other.

\subsection{Minor Clashes}

Figure 4 represents a minor clash workflow. After encountering a clash, the contractor's engineering team is searching for a solution. The next step is to find out about availability of materials. If all materials are available, it is possible to implement the solution if not it is necessary to wait for the delivery. Based on the worst scenario the time equation could be as below.

$$
T^{c}=T^{s s}+T^{d}+T^{w}
$$

where $T^{c}$ is the total duration of a clash, $T^{s s}$ is the time for which engineering team is searching for a solution, $T^{d}$ is the waiting time for delivery and $T^{w}$ is the time necessary for implementing the solution.

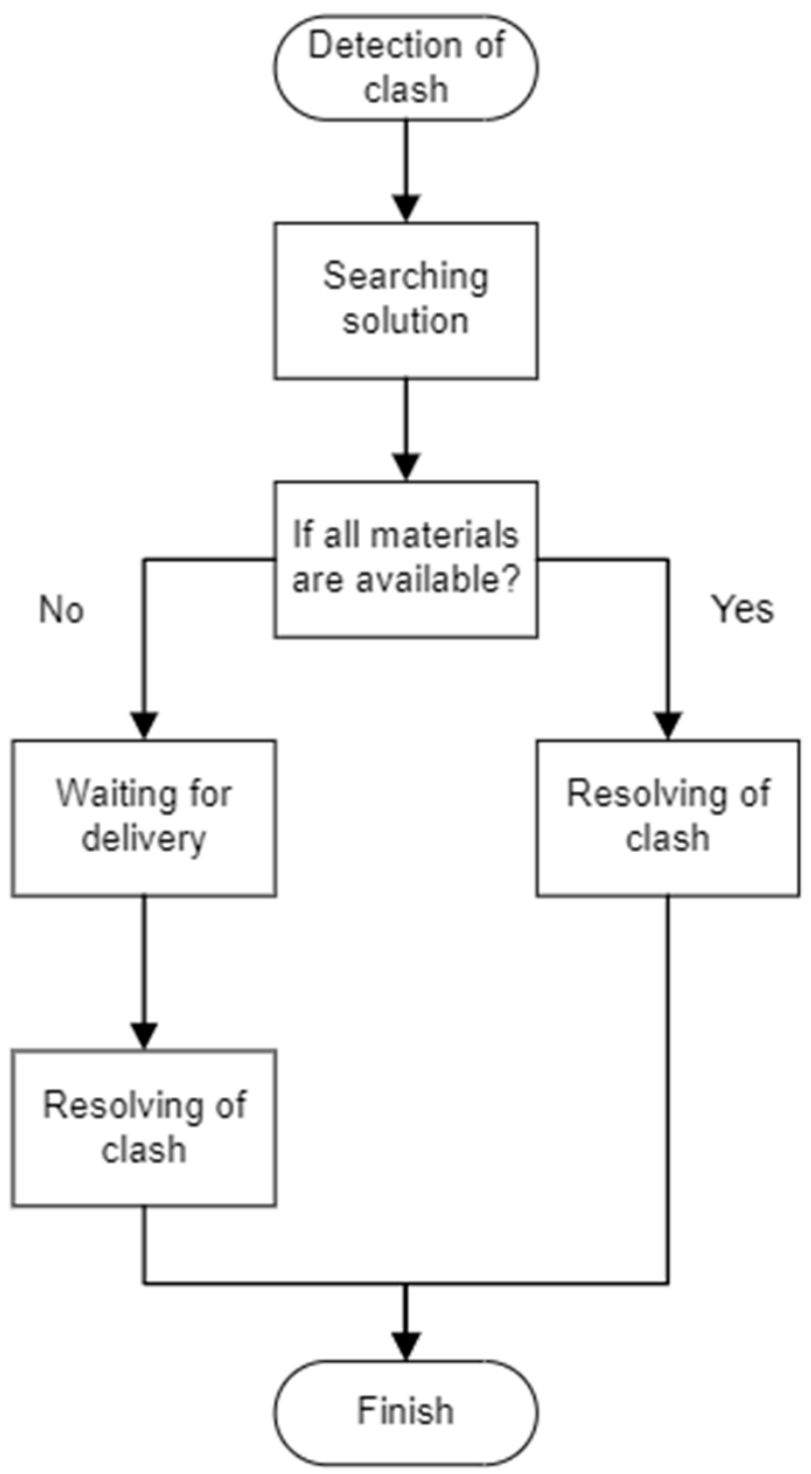

Figure 4. Minor clash workflow. 
Using BIM, two out of three factors from the Equation (1) would be eliminated. $T^{\mathcal{S S}}$ and $T^{d}$ would be moved into the pre-construction stage. The formula has changed and look as follows.

$$
T^{c b i m}=T^{w}
$$

where $T^{c b i m}$ is the total duration of clash using BIM.

Total cost of clash which is detected and resolved during the construction stage is presented below.

$$
C^{c}=\left(U C^{s s} \times T^{s s}\right)+C^{d}\left(U C^{p} \times T^{c}\right)
$$

where $C^{c}$ is a total clash cost, $U C^{s s}$ is the unity cost of engineering work, $C^{d}$ is the cost of materials, $U C^{p}$ is the unity cost of blue collar work.

Using BIM, the overall cost of engineering team is eliminated and moved into the project stage. Moreover, the total cost of blue collar work will be reduced. In Equation (3) this cost is dependent on three times: $T^{s s}, T^{d}$ and $T^{w}$. With BIM it will be only $T^{w}$. The changed formula will be as follows.

$$
C^{c b i m}=C^{d}+\left(U C^{p} \times T^{w}\right)
$$

where $C^{c b i m}$ is the total clash cost with BIM.

\subsection{Medium Clash}

Medium clashes demand more changes than minor ones, therefore, consultations between contractor and designer could be required. Figure 5 depicts a medium clash workflow. The first step is to find a solution by the contractor's engineering team. Afterwards, that solution is consulted with the designer. If there is agreement, the work can move on, if not, the contractor and designer cooperate together to find a proper resolution. If the work is fairly advanced and the solution requires large changes, disassembly will be needed. The remaining steps are the same as in the minor clash. Based on all this information, the medium clash time Equation is as follows.

$$
T^{\mathcal{c}}=T^{s \mathcal{S}}+T^{c o}+T^{d i}+T^{d}+T^{w}
$$

where $T^{c}$ is the total duration of a clash, $T^{s S}$ is the time in which engineering team is searching for a solution, $T^{c o}$ is the time of the consultations between contractor and designer, $T^{d i}$ is the time necessary for carrying out the dismantling, $T^{d}$ is a waiting time for delivery and $T^{w}$ is the time necessary for implementing the solution.

Using BIM, total clash duration would be as in Equation (2). Factors $T^{s s}, T^{c o}$ and $T^{d}$ would be moved into the pre-construction stage while $T^{d i}$ would be non-existent from a lack of need for dismantling.

Total cost of medium clash is presented below.

$$
C^{c}=\left(U C^{s s} \times\left(T^{s S}+T^{c o}\right)\right)+C^{d i}+C^{d}+\left(U C^{p} \times T^{c}\right)
$$

where $C^{c}$ is the total clash cost, $U C^{s s}$ is the unity cost of engineering work, $C^{d i}$ is the cost of materials which could not be used after dismantling, $C^{d}$ is the cost of materials, $U C^{p}$ is the unity cost of blue collar work.

In that case compared to a minor clash, the cost of engineering work is increased because of multiplying $U C^{s s}$ by $T^{s s}$ and $T^{c o}$ not only $T^{s s}$. However, using BIM it is meaningless on the construction stage, because finding a solution of clash is an assignment for designers. Another important cost which is eliminated by BIM is the cost of wasted materials. Owing to having a clash resolution on the pre-construction stage there will be no need of conducting demolitions. Moreover, cost of labor will be reduced. Overall medium clash costs will be the same as as minor clashes. 


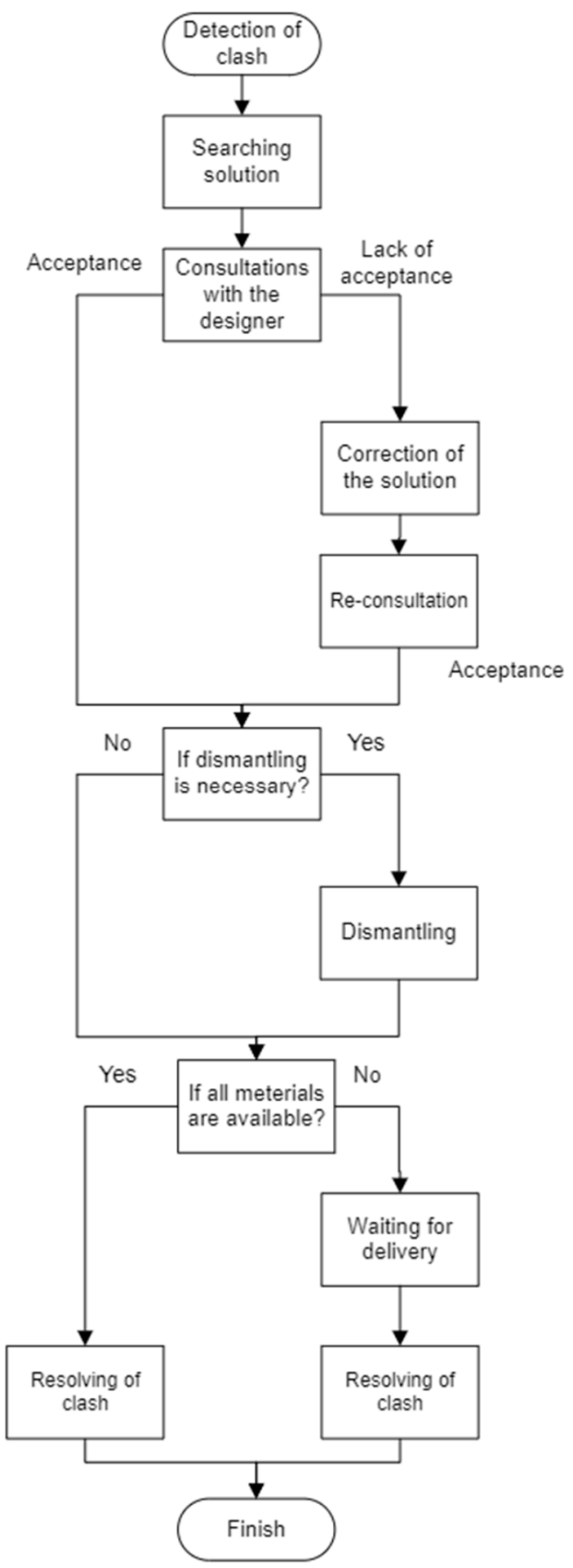

Figure 5. Medium clash workflow.

\subsection{Major Clash}

Major clashes involve the largest number of stakeholders and cause the biggest financial losses. In this case it might be necessary to rebuild not only two colliding installations, but also some of the surrounding elements. Such actions could interfere with the functional and visual characteristics of the building; therefore, the clients' approval would be required. Major clash workflow is presented on Figure 6. 


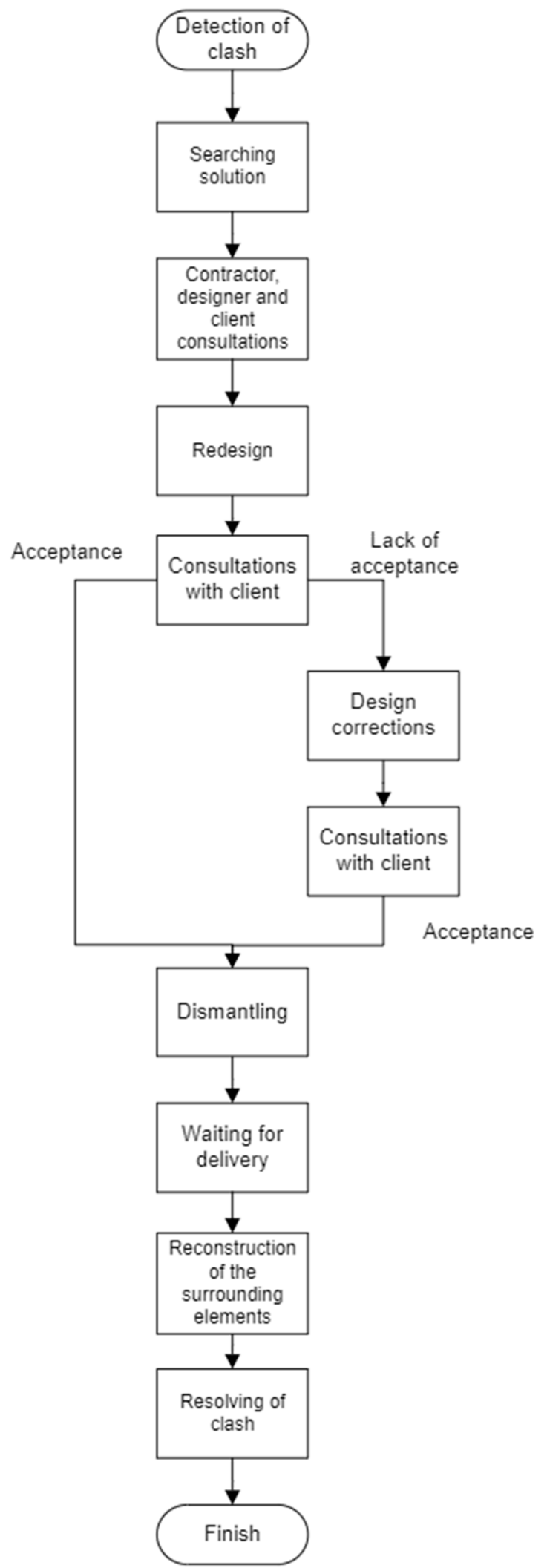

Figure 6. Major clash workflow.

Because it is usually unknown at the beginning which actions should be introduced to resolve a clash, the contractors' engineering team works to find a solution. After they realize that it is impossible to resolve a clash without making project changes it comes 
down to contractor, designer and client consultations. The project is given to the designer for redesign. Further consultations are carried out until the client's approval is given. It is important to point out that these deliberations could take a huge amount of time because of difficult negotiations in which stakeholders try to come to terms with who should cover the costs of redesign and reconstruction. When the agreement is finished, elements which are involved in changes could be dismantled. At almost the same time the deliveries are made. After that, the infrastructure elements which are involved in the changes are rebuilt, the clash is resolved and the construction work can move on. Based on that the time equation will be as following.

$$
T^{\mathcal{c}}=T^{s \mathcal{S}}+T^{c 1}+T^{r}+T^{c 2}+T^{d i}+T^{d}+T^{w}
$$

where $T^{c}$ is the total duration of a clash, $T^{c 1}$ is the time for which stakeholders discuss various scenarios and when the decision about redesign is made, $T^{r}$ is the redesign time, $T^{c 2}$ is the time of consultations with client, $T^{s s}$ is the time in which engineering team is searching for a solution, $T^{c o}$ is time of consultations between contractor and designer, $T^{d i}$ is the time necessary for carrying out the dismantling, $T^{d}$ is the waiting time for delivery and $T^{w}$ is the time necessary for implementing the solution.

Using BIM the majority of consultations with client and design changes would be carried out in the pre-construction stage. The dismantling and delays in deliveries also would not be present. Therefore, in that case as in the previous ones, all components except $T^{w}$ would not be involved, so a BIM clash time formula would be as in (2).

Total major clash cost formula without using BIM is presented below.

$$
C^{c}=\left(U C^{s s} \times T^{s s}+\left(U C^{r} \times\left(T^{c 1}+T^{c 2}+T^{r}\right)\right)+C^{d i}+C^{d}+\left(U C^{p} \times T^{c}\right)+C^{p e n}\right.
$$

where $C^{c}$ is the total clash cost, $U C^{S S}$ is the unity cost of engineering work, $U C^{r}$ is the unity cost of the designer's work, $C^{d i}$ is the cost of materials which could not be used after dismantling, $C^{d}$ is the cost of materials, $U C^{p}$ is the unity cost of blue collar work, $C^{p e n}$ is the cost of penalties for delays.

In major clash case, usage of BIM moves the cost of searching for a solution and redesign into the pre-construction stage. The cost of dismantled elements is removed and the cost of blue collar work is minimized. Another important element which is removed is the cost of penalties for delays. It is a common cost which could occur in the case of a major clash and cause a lot of financial losses. According to all the information presented above, BIM clash costs can be described by Equation (4).

\section{Conclusions}

All engineers that work on the project are dealing with a dynamic field. This requires a very effective coordination and collaboration between many stakeholders to obtain the desired result. The dynamic field of any design project involves many stakeholders such as structural engineers, architects, contractors, suppliers and vendors. This makes the project completion process highly complex. BIM clash detection is used as one of the most efficient tools for virtual work coordination. Nowadays it should be integrated to the BIM model processing workflow. The key benefit of BIM methodology is the possibility of detecting installation collisions for reducing the cost of the building stage.

Collisions are the main factor forcing contractors to incur additional expenditures. The aim of this study was to check how they affect the unforeseen costs of a construction investment and how the building information modeling methodology can help to reduce them. For this purpose, the collisions were divided into three categories-minor, medium and major. For each of them, a diagram has been created that describes the steps leading to its solution. These diagrams served as the basis for the theoretical determination of the time and financial outlays needed to remove the collision. The analysis showed that the cost of each version of a collision has a different number of components. The application of the building information modeling methodology brings the expenses related to all three types 
of collisions to one form. Of course, the expenses incurred will, of course, differ from each other, because each case is different, takes a different amount of time, different materials, or greater or lesser human resources. Nevertheless, thanks to locating and resolving collisions before the construction stage, expenses related to demolition, design changes, financial penalties or indirect costs related to the need to pay for personnel and equipment during work stoppages are eliminated.

This article provides a strong theoretical basis for the economic and technical analysis of the costs of construction collisions, with particular emphasis on electrical installations. The above considerations should be confirmed by empirical research. In Poland, a significant part of investments is carried out in the traditional system, and only a part (e.g., model of an underground garage with installations) is modeled using BIM at the stage of construction. So, it is possible to compare traditional 2D technology with BIM.

Author Contributions: Conceptualization, T.D. and M.O.; methodology, T.D. and M.O.; software, M.O.; validation, T.D., M.O. and A.S.; formal analysis, M.O.; investigation, T.D. and M.O.; resources, T.D. and M.O.; writing—original draft preparation, T.D.; writing—review and editing, A.S.; visualization, M.O.; supervision, T.D.; project administration, T.D. All authors have read and agreed to the published version of the manuscript.

Funding: This research received no external funding.

Institutional Review Board Statement: Not applicable.

Informed Consent Statement: Not applicable.

Data Availability Statement: Not applicable.

Conflicts of Interest: The authors declare no conflict of interest.

\section{References}

1. Barlish, K.; Sullivan, K. How to measure the benefits of BIM-A case study approach. Autom. Constr. 2012, 24, 149-159. [CrossRef]

2. Woo, J.; Wilsmann, J.; Kang, D. Use of as-built building information modeling. Constr. Res. Congr. 2010, 1, $538-547$.

3. Bryde, D.; Broquetas, M.; Volm, J.M. The project benefits of Building Information Modelling (BIM). Int. J. Proj. Manag. 2013, 31, 971-980. [CrossRef]

4. El Saddik, A. Digital twins: The convergence of multimedia technologies. IEEE MultiMedia 2018, 25, 87-92. [CrossRef]

5. Chahrour, R.; Hafeez, M.A.; Ahmad, A.M.; Sulieman, H.I.; Dawood, H.; Rodriguez-Trejo, S.; Kassem, M.; Naji, K.K.; Dawood, N. Cost-benefit analysis of BIM-enabled design clash detection and resolution. Constr. Manag. Econ. 2021, 39, 55-72. [CrossRef]

6. Ostapowski, M. Collision Cost Analysis Based on BIM Technologies. Master's Thesis, Warsaw University of Technology, Warsaw, Poland, 2021.

7. Matejka, P.; Sabart, D. Categorization of clashes and their impacts on Construction projects. In Proceedings of the 17th International Scientific Conference Engineering for Rural Development, Jelgava, Latvia, 23-25 May 2018.

8. Althizer, J. The Financial Benefit of Using Technology for Clash Reduction, Hourigan, 22 July. Available online: https://www. hourigan.group/blog/financial-benefit-using-technology-clash-reduction (accessed on 6 March 2021).

9. Akponeware, A.O.; Adamu, A.A. Clash Detection or Clash Avoidance? An Investigation into Coordination Problems in 3D BIM. Buildings 2017, 7, 75. [CrossRef]

10. Azhar, S. Building Information Modeling (BIM): Trends, Benefits, Risks, and Challenges for the AEC Industry. Leadersh. Manag. Eng. 2011, 11, 241-252. [CrossRef]

11. Zhang, J.P.; Hu, Z.Z. BIM- and 4D-based integrated solution of analysis and management for conflicts and structural safety problems during construction: 1. Principles and methodologies. Autom. Constr. 2011, 20, 155-166. [CrossRef]

12. Wang, J.; Wang, X.; Shou, W.; Chong, H.Y.; Guo, J. Building information modeling-based integration of MEP layout designs and constructability. Autom. Constr. 2016, 61, 134-146. [CrossRef]

13. Pärn, E.A.; Edwards, D.J.; Sing, M.C.P. Origins and probabilities of MEP and structural design clashes within a federated BIM model. Autom. Constr. 2018, 85, 209-219. [CrossRef]

14. Lefevre, M. Build Smarter, Faster, and Cheaper with BIM. Available online: http://www.buildings.com/article-details/articleid/ 6149 /title/build-smarter-faster-and-cheaperwith-bim (accessed on 17 March 2021).

15. Neelamkavil, J.; Ahamed, S.S. The Return on Investment from BIM-Driven Projects in Construction, NRC Publications Archive, Research Report; National Research Council of Canada, Institute for Research in Construction: Ottawa, ON, Canada, 2012.

16. Methodology for Cost Benefit Analysis for the Use of BIM in Public Tenders. Available online: https:/ / ec.europa.eu/easme/en/ tenders/methodology-cost-benefit-analysis-use-bim-public-tenders (accessed on 17 March 2021). 